\title{
Synthesis and properties of faceted hexagonal hydroxyapatite nanotubes
}

\author{
Tingting $\mathrm{Li}^{1, \mathrm{a}}$, Ziqiang Gong ${ }^{1, \mathrm{~b}}$, Haiying Dong ${ }^{2, \mathrm{c}_{*}}$ and Xiaoyun $\mathrm{Li}^{1, \mathrm{~d}_{*}}$ \\ ${ }^{1}$ Center for Nanoscience and Nanotechnology, Zhejiang Sci-Tech University, \\ Hangzhou 310018, China \\ ${ }^{2}$ Ningde Hospital affiliated to Fujian Medical University, Ningde 352000, China

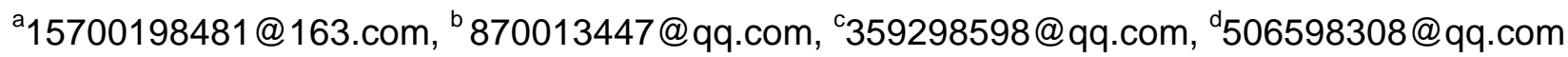

Keywords: hydroxyapatite, nanorod, nanotube, one-step hydrothermal

Abstract. Nanoscale hydroxyapatite (HA) is an optimal candidate biomaterial for bone tissue engineering because of its excellent bioactive and osteoconductive properties. Here we have been successfully synthesized the faceted hexagonal hydroxyapatite nanotubes by one-step hydrothermal method. The obtained product microstructure is nanotube with faceted hexagonal. The results of cell culture shows that those novel HA nanatubes are nontoxic for the proliferation of the cell, which gives a great potential for bone repairation.

\section{Introduction}

Hydroxyapatite $\left(\mathrm{Ca}_{10}\left(\mathrm{PO}_{4}\right)_{6}(\mathrm{OH})_{2}\right)$, a synthetic bioceramic, as the main inorganic mineral constituent of human bone and teeth, has attracted extensive attention in biomedical applications because of its brilliant biocompatibility and osteoconductivity $[1,2]$. In biological materials, form and function are intimately related, which may affect its performance significantly. Hence there has been great interest in developing new strategies to fabricate novel morphology for out-bound performance [3].

In the past couple of years, several methods have been developed to fabricate HA with a varieties of morphology and structure to improve its bio-performance of certain aspects. One-dimensional nano-structured materials, such as nanowires and nanotubes, have attracted great attention because of their restricted size and high surface area for bio-applications [4, 5]. Hydroxyapatite nanotubes tubular structure similar to cancellous bone, is conducive to bone tissue and blood vessel ingrowth, what's more, high surface area is conducive to biodegradation. However, up to date, most of these previous studies have been reported mainly on spheric, cubic, or cylindrical HA, fewer studies have focused on the HA nanotube due to lack of synthesis methods for such materials [4]. In this article, we described an unprecedented route to synthesis impurity-free HA nanotube with hexagonal facets.

\section{Experimental}

Synthesis of HA nanotube. All reagents used in the synthesis were of analytical grade and purchased from Hangzhou Gaojing Fine Chemical Industry Co., Ltd. without further purification. Firstly, 0.33g $\mathrm{CaCl}_{2}$ and $0.319 \mathrm{~g} \mathrm{NaH} \mathrm{PO}_{2} \cdot 2 \mathrm{H}_{2} \mathrm{O}$ were dissolved in two $25 \mathrm{~mL}$ mixed solution of deionized water and ethylene glycol (EG) with equal volume ratio respectively. Secondly, $\mathrm{NaH}_{2} \mathrm{PO}_{4} \cdot 2 \mathrm{H}_{2} \mathrm{O}$ solution was slowly added into the $\mathrm{CaCl}_{2}$ solution at $1.0 \mathrm{~mL} \cdot \mathrm{min}^{-1}$. Thirdly, $50 \mathrm{ml} \mathrm{N}, \mathrm{N}$ - dimethylacetamide (DMAC) was added into above mixed solution at $1.0 \mathrm{~mL} \cdot \mathrm{min}^{-1}$ respectively, the final mixed solvents (H2O/EG/DMAC) with the ratio of 1:1:2. Finally, the obtained suspension was transferred into a $30 \mathrm{~mL}$ sealed Teflon-lined stainless steel autoclave and heated under $220^{\circ} \mathrm{C}$ for $4,8,12 \mathrm{~h}$. The product was separated by centrifugation and washed with deionized water, and dried at $60^{\circ} \mathrm{C}$ for further characterization.

Characterization. Morphology and structure of the HA nanotube was characterized via a field emission scanning electron microscope (FESEM; HITACHI S-4800, Japan) and JEOL JEM 2010 transmission electron microscopy (TEM). X-ray diffraction (XRD) analysis was performed on a Bruker D8-discover instrument operating with $\mathrm{Cu} \mathrm{Ka}$ radiation $(\lambda=1.5406 \AA)$. 
Cell Culture and Treatment. The powder of HA nanotube was Co-cultured with MG63 cells for 2,4 and 6 days. The dose of the hydroxyapatite is $100 \mathrm{mg} / \mathrm{L}$. In control experiments, no hydroxyapatite power but culture medium was added. The cells on the samples were fixed and permeabilized for fluorescence imaging. Cell viability was evaluated by using a 3-(4,5-dimethylthiazol-2-yl)-2,5-diphenyl tetrazolium bromide (MTT; Sigma) reduction conversion assay and cell survival was expressed as absorbance at $570 \mathrm{~nm}$ in a spectrophotometric microplate reader (Bio-RAD Model 680, USA). All data reported in this article were the mean value of 3 examinations [6].

\section{Results and discussions}

Typical electron microscopy images of the as-prepared product are shown in Fig. 1a-d. When the mixed solvents $(\mathrm{H} 2 \mathrm{O} / \mathrm{EG} / \mathrm{DMAC})$ with the ratio of $1: 1: 2$ and the reaction temperature was $220^{\circ} \mathrm{C}$, the prepared product is nanorods (Fig. 1a). When the reaction hour raised to $8 \mathrm{~h}$, the products began to open at the endpoint (Fig. 1b), the products are completely transformed into nanotubes (Fig. 1c) with the reaction time increased to $12 \mathrm{~h}$. Fig. $1 \mathrm{~d}$ is the TEM images of HA nanotubes, we can see the internal structure of tubular from the figure. On the basis of the morphological evolution of the time-dependent experiment results, the intrinsic growth mechanisms of the HA nanotube were proposed (Scheme 1). This scheme shows the process of morphology transformation of the hydroxyapatite during the hydrothermal process. XRD profiles of the products obtained are shown in Fig. 2. XRD pattern confirms the main component of preparing sample is HA, in Fig. 2 the diffraction characteristic peaks at $2 \theta=25.9^{\circ}, 31.8^{\circ}, 32.2^{\circ}$ and $32.9^{\circ}$ are assigned to the (002), (211), (112) and (300) planes of HA crystal.
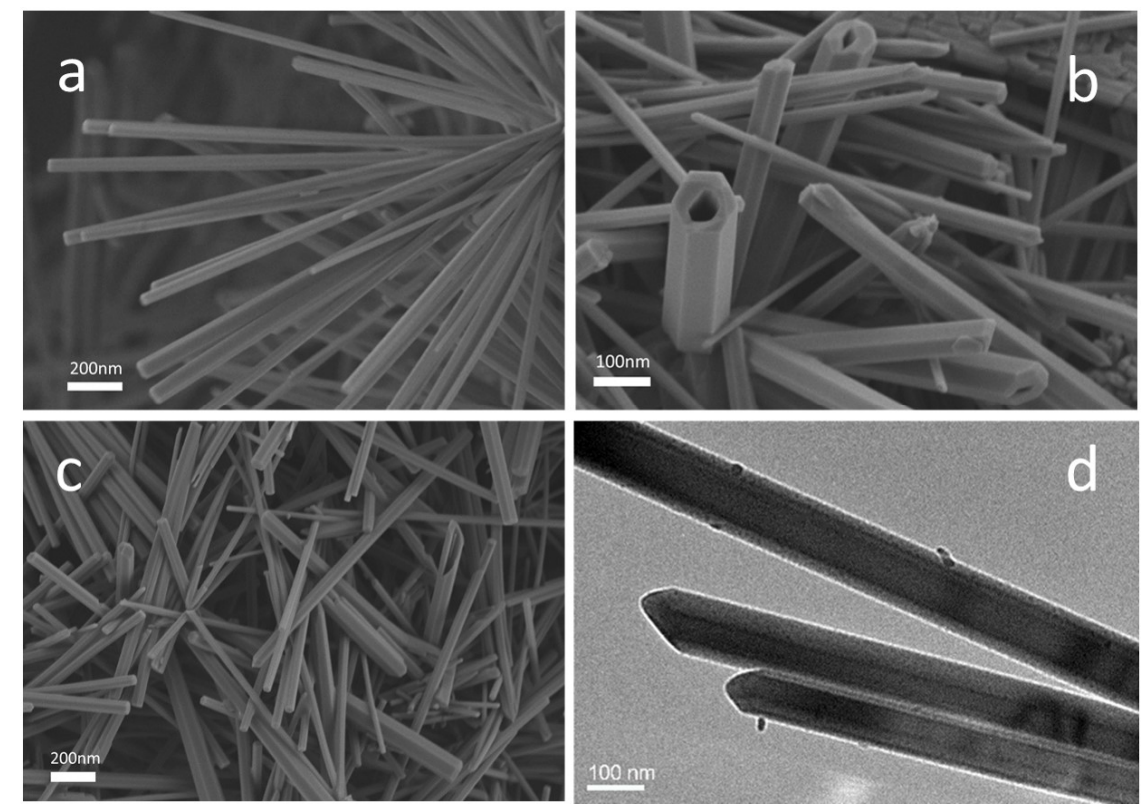

Figure 1, SEM (a-c) and TEM (d) images of prepared HA nanotubes

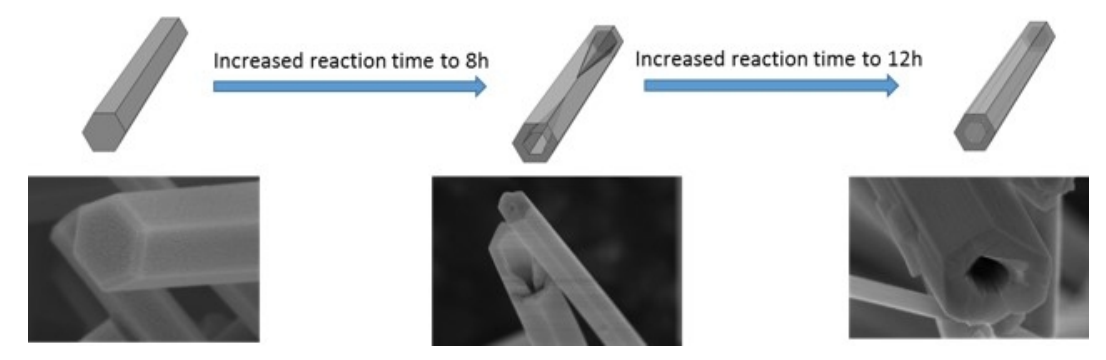

Scheme 1. Schematic demonstration of the preparation of the HA nanotube 


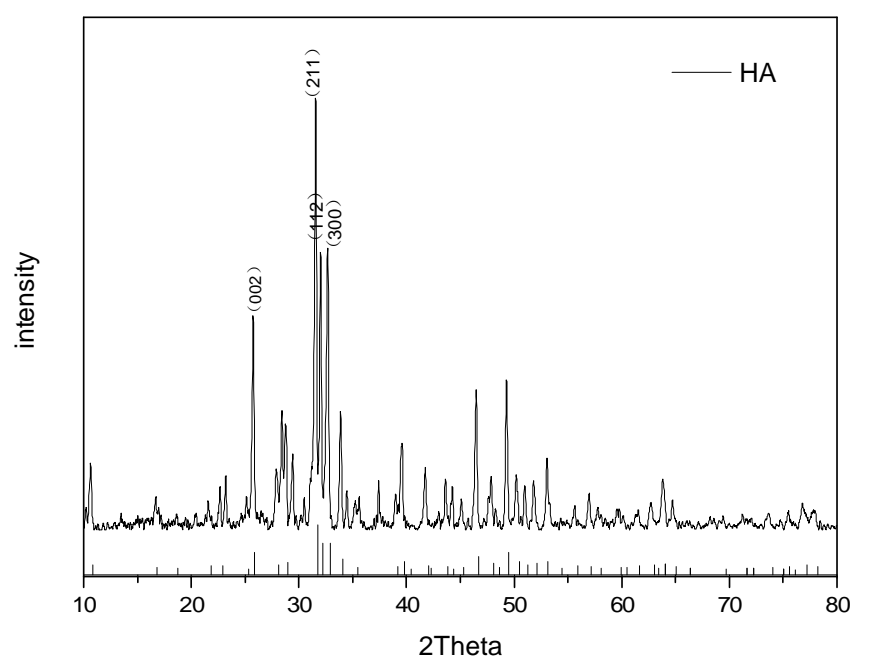

Figure 2. XRD patterns of HA product

It has been shown that an interplay exists between osteoblasts and nanoapatite features. Here we culture the osteoblasts with HA obtained in vitro to investigate the effects of the prepared HA on cell proliferation and migration [6]. The results of the 6d cell culture in vitro are shown in Fig. 3 and Fig. 4. The fluorescence microscopy images of live cells Co-culture with HA were shown in Fig.3. The live cells appear as viridity spots and related cytoplast as purplish blue spots. From the cell proliferation bar charts (Fig. 4), we can see the product boost the cell proliferation to a certain extent.
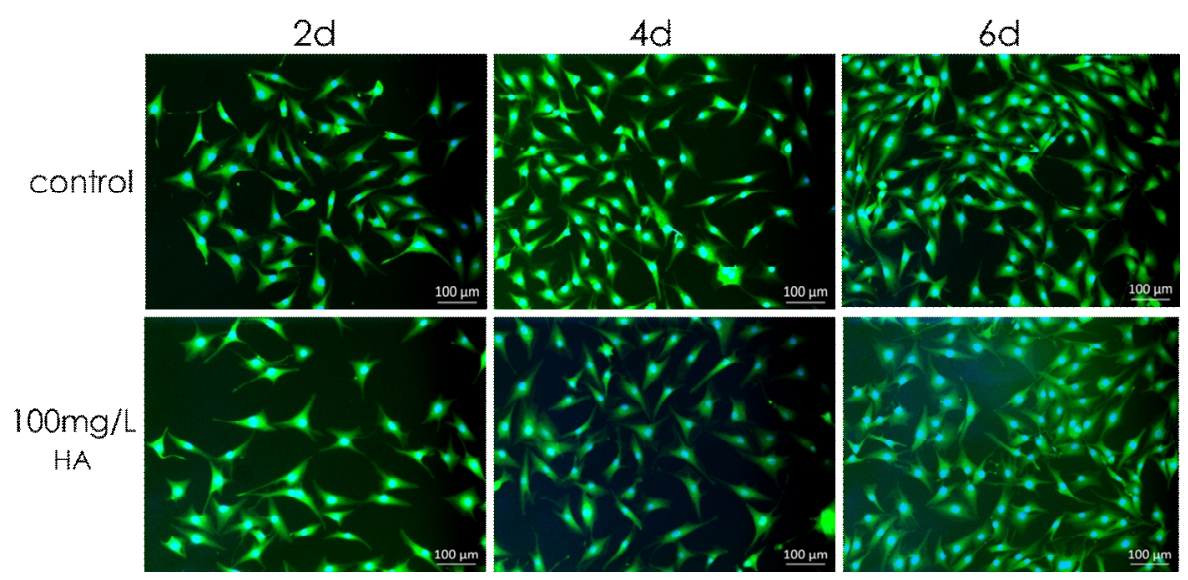

Figure 3. Fluorescence microscopy images of MG63 cells were co-cultured with HA nanotube for 2, 4, 6 days 


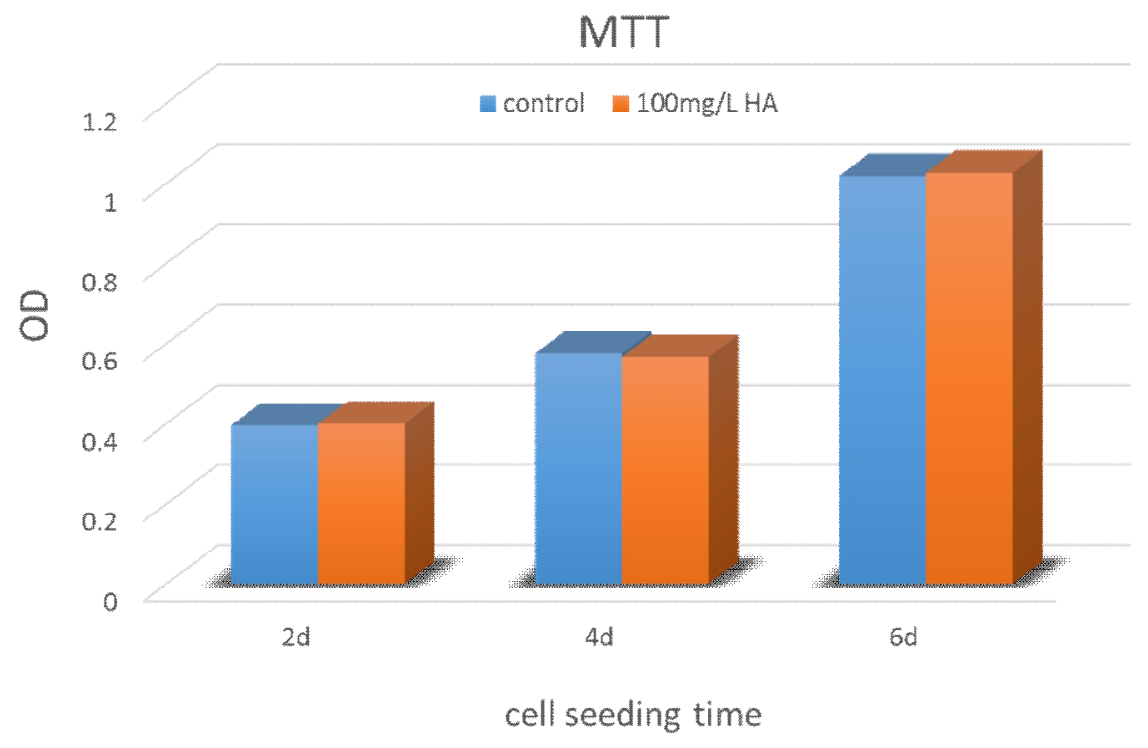

Figure 4. MTT of MG63 cells were co-cultured with HA nanotube for 2, 4 and 6 days culture

\section{Conclusions}

In this paper, we develop an facile route to synthesis hexagonal faces HA nanotubes. The synthesis does not need any template or surfactant, avoiding the complicated procedures and cost for their removal in the product. What's more, their nanotube stucture is conducive to bone tissue and blood vessel ingrowth and high surface area is conducive to biodegradation and the results of cell culture shows that those novel HA nanatubes are nontoxic for the proliferation of the cell, which gives it great potential for bone repairation.

\section{References}

[1] Costa D O, Dixon S J, Rizkalla A S. One-and three-dimensional growth of hydroxyapatite nanowires during sol-gel-hydrothermal synthesis[J]. ACS applied materials \& interfaces, 2012, 4(3): 1490-1499.

[2] Guo X, Yu L, Chen L, et al. Organoamine-assisted biomimetic synthesis of faceted hexagonal hydroxyapatite nanotubes with prominent stimulation activity for osteoblast proliferation[J]. Journal of Materials Chemistry B, 2014, 2(13): 1760-1763.

[3] Liu G, Zhao D, Tomsia A P, et al. Three-dimensional biomimetic mineralization of dense hydrogel templates[J]. Journal of the American Chemical Society, 2009, 131(29): 9937-9939.

[4] Yuan Y, Liu C, Zhang Y, et al. Sol-gel auto-combustion synthesis of hydroxyapatite nanotubes array in porous alumina template[J]. Materials Chemistry and Physics, 2008, 112(1): 275-280.

[5] Yang Z, Huang Y, Chen S T, et al. Template synthesis of highly ordered hydroxyapatite nanowire arrays[J]. Journal of materials science, 2005, 40(5): 1121-1125.

[6] Zhao H, Dong W, Zheng Y, et al. The structural and biological properties of hydroxyapatite-modified titanate nanowire scaffolds[J]. Biomaterials, 2011, 32(25): 5837-5846. 